\title{
Câncer de Pele em Pescadores: Evidências Científicas para o Cuidado em Saúde
}

\author{
Skin Cancer in Fishermen: Scientific Evidence for Health Care
}

\author{
Joabson Romário Borges Mendonça*a; Francisco Arlyson da Silva Veríssimo ${ }^{\mathrm{b}}$
}

${ }^{a}$ Escola de Saúde Pública do Ceará. CE, Brasil.

${ }^{b}$ Faculdade Princesa do Oeste. CE, Brasil.

*E-mail: joabsonromario@gmail.com

\begin{abstract}
Resumo
O câncer se constitui, atualmente, como uma das patologias mais prevalentes em todo o Mundo, destacando-se o câncer de pele. Na realidade brasileira, o número de casos novos de câncer de pele do tipo não melanoma para cada ano, do triênio 2020-2022, será de 83.770 casos em homens e de 93.160 em mulheres, correspondendo a um risco estimado de 80,12 casos novos a cada 100 mil homens e 86,65 casos novos a cada 100 mil mulheres. Objetiva-se por meio deste estudo revisar a literatura a respeito do câncer de pele em pescadores. Trata-se de uma revisão integrativa ocorrida entre os meses de agosto a dezembro de 2020, utilizando-se as bases de dados: Scielo, BDENF, Medline e Lilacs, com total de cinco estudos selecionados. Como palavras-chave foram eleitos: "Câncer de Pele", "Pesca", "Promoção de Saúde". Constata-se a preponderância da influência dos fatores ocupacionais para o adoecimento, visto que determinadas profissões expõem seus trabalhadores à radiação solar sem fatores de proteção, convertendo-os em mais sensíveis a essa problemática. Os pescadores, por seu labor diário, bem como por suas características sociodemográficas, organizacionais e culturais se tornam mais susceptíveis ao risco de desenvolver este tipo de neoplasia. Esta patologia apresenta taxa de morbimortalidade considerável, além de gerar afastamento do trabalho, causando déficits na economia familiar. Outrossim, sabe-se que a doença é passível de prevenção, sobretudo com utilização de medidas simples e acessíveis.
\end{abstract}

Palavras-chave: Pesca. Câncer de Pele. Cuidados Preventivos. Promoção da Saúde.

\begin{abstract}
Cancer is currently one of the most prevalent pathologies worldwide, and skin cancer standing out among them. In the Brazilian reality, the number of new cases of non-melanoma skin cancer expected, for each year of the 2020-2022 triennium, will be 83,770 cases in men and 93,160 in women, corresponding to an estimated risk of 80.12 cases new per 100,000 men and 86.65 new cases per 100,000 women. The objective of this study is to review the literature on skin cancer in fishermen. This is an integrative review that took place between August and December 2020, using the databases Scielo, BDENF, Medline and Lilacs, with a total of 05 selected studies. As keywords, "Skin Cancer", "Fishing", "Health Promotion" were chosen. There is a preponderance of the occupational factors influence for illness, since certain professions expose their workers to solar radiation without protective factors, making them more sensitive to this problem. Fishermen, due to their daily work, as well as their sociodemographic, organizational and cultural characteristics, become more susceptible to the risk of developing this type of neoplasia. This pathology has a considerable morbidity and mortality rate, in addition to causing absence from work, causing deficits in the family economy. Furthermore, it is known that the disease is preventable, especially with the use of simple and accessible measures.
\end{abstract}

Keywords: Fishermen. Skin Cancer. Preventive Health Care. Health Promotion.

\section{Introdução}

O câncer se constitui como um dos mais sérios problemas de Saúde Pública em todo o Mundo, e está entre as quatro principais causas de morte prematura (antes dos 70 anos de idade) (OPAS, 2013). A taxa de incidência é maior em países desenvolvidos, porém é nos países de baixa e média renda em que acontece cerca de $70 \%$ das mortes. Nestes se observam ainda mortalidade precoce e dificuldades para acessar meios de prevenção. É uma patologia de origem multifatorial, resultante, principalmente, de fatores genéticos, exposição a condicionantes ambientais e estilo de vida (BRAY et al., 2018).

Conforme o Instituto Nacional do Câncer (INCA, 2019), o câncer se caracteriza como um conjunto de mais de cem doenças, que tem como gênese o crescimento acelerado e desordenado de células. Tal processo tende a ser muito agressivo e incontrolável, determinando a formação de tumores malignos que podem se disseminar para diversas regiões do corpo, aumentando assim o risco de morte. Os principais órgãos acometidos por câncer são: pulmão, mama, colorretal, próstata, estômago e pele.

Entre os tumores de pele, o tipo não-melanoma é o de maior incidência e mais baixa letalidade, sendo mais comum em pessoas com mais de 40 anos. Divide-se em carcinomas basocelulares e espinocelulares. O tipo melanoma é menos frequente, porém tem o pior prognóstico e mais alto índice de mortalidade (ACS, 2019).

Os sinais e sintomas mais comuns relacionadas ao câncer da pele são: manchas que coçam, doem, sangram facilmente ou descamam; lesões de pele que não cicatrizam em quatro semanas; sinal que muda de cor, textura, tamanho, espessura ou contornos; elevação ou nódulo circunscrito e adquirido 
da pele que aumenta de tamanho e tem aparência perolada, translúcida, avermelhada, castanha ou escura (INCA, 2019).

No ano de 2018, em todo o Mundo, foram estimados 1,04 milhões $(5,8 \%)$ de casos novos de câncer de pele nãomelanoma, com 640 mil casos novos em homens (16,6/100 mil) e 400 mil casos novos em mulheres (10,7/100 mil). As maiores taxas de incidência do câncer de pele não-melanoma estão na Austrália e Nova Zelândia, América do Norte e nos países da Europa Ocidental tanto para homens quanto para as mulheres. Para o câncer de pele melanoma, as maiores incidências estão na Austrália e Nova Zelândia e nos países do Norte, Centro e Leste Europeu (FERLAY et al., 2018).

Estima-se que tal aumento na mortalidade por câncer nestes países desenvolvidos ocorre em função do envelhecimento e crescimento populacional e as mudanças nos fatores de riscos, consequentes da realidade socioeconômica em que se inscrevem. Nesse ínterim, observa-se uma mudança na tipologia desses cânceres, associando-se bem mais a hábitos e estilo de vida (sedentarismo, alimentação inadequada) do que a fatores infecciosos, mais comuns em países mais pobres (BRAY et al., 2018).

$\mathrm{Na}$ realidade brasileira, o número de casos novos de câncer de pele não melanoma esperados, para cada ano, do triênio 2020-2022, será de 83.770 em homens e de 93.160 em mulheres, correspondendo a um risco estimado de 80,12 casos novos a cada 100 mil homens e 86,65 casos novos a cada 100 mil mulheres (INCA, 2019).

$\mathrm{O}$ câncer de pele não-melanoma em homens é mais incidente nas Regiões Sul, Centro-Oeste e Sudeste, com um risco estimado de 123,67/100 mil, 89,68/100 mil e 85,55/100 mil, respectivamente. Nas Regiões Nordeste e Norte, ocupa a segunda posição, com um risco estimado de 65,59/100 mil e 21,28/100 mil, respectivamente (IBGE, 2019).

Quanto ao câncer de pele melanoma, o número de casos novos estimados será de 4.200 em homens e de $4.250 \mathrm{em}$ mulheres. Esses valores correspondem a um risco estimado de 4,03 casos novos a cada 100 mil homens e 3,94 para cada 100 mil mulheres. Os números estimados para 2020 no Ceará apontam 120 novos casos de câncer de pele melanoma, sendo que para Fortaleza serão 40 novos casos (INCA, 2019).

É importante relatar que a exposição solar contribui, de forma decisiva, para a ocorrência de cânceres de pele, sendo que a maioria desses cânceres se desenvolvem em regiões corporais mais expostas ao Sol. Os caucasianos têm maior risco para câncer de pele quando comparados a pessoas de pele negra. Também se cita que certas profissões são mais sensíveis à tal adoecimento, pois se expõem ao Sol por mais horas e sem fatores de proteção, destacando-se agricultores, trabalhadores da construção civil, mineração e pescadores (BRASIL, 2012).

Os pescadores são trabalhadores que se utilizam da pesca para sua subsistência: alimentam-se e comercializam os excedentes. Trabalham de forma autônoma ou em regime de economia familiar, com meios de produção próprios ou mediante contrato de parcerias. Normalmente, essa atividade é passada de geração em geração, isto é, os filhos tendem a reproduzir o fazer de seus pais. A classe ainda se baseia na informalidade do trabalho, o que gera maiores riscos ocupacionais. Economicamente, são responsáveis por $65 \%$ da pesca extrativista no Brasil - são 550 mil toneladas por ano. Em termos de produção nacional, considerando a aquicultura, a pesca artesanal representa cerca de $50 \%$ do total de pescados produzidos no país (RIOS, 2011).

Assim, o objetivo deste trabalho é analisar a produção científica acerca do Câncer de Pele em Pescadores, ao identificar estudos que discorram sobre os fatores de riscos para a ocorrência de câncer de pele em pescadores.

\section{Desenvolvimento}

\subsection{Metodologia}

O presente estudo se apresenta como uma revisão integrativa da literatura de caráter qualitativo, descritivo que discorre sobre a temática "Câncer de Pele em pescadores". A revisão integrativa permite definir um conhecimento atual sobre uma temática específica, já que é conduzida de modo a identificar, analisar e sintetizar resultados de estudos independentes sobre o mesmo assunto, contribuindo para uma possível repercussão benéfica na qualidade dos cuidados prestados ao paciente.

Este tipo de estudo é um método que inclui a análise de pesquisas relevantes que dão suporte para a tomada de decisão e melhoria da prática clínica. Dessa forma, possibilita a síntese do estado de conhecimento de um determinado assunto, além de apontar lacunas do conhecimento que precisam ser preenchidas com a realização de novos estudos. Por sua vez, consiste em uma abordagem mais ampla, podendo incluir estudos experimentais e não experimentais; a combinação de dados da literatura empírica e teórica, gerando assim, uma ampla amostra (GALVÃO, 2004).

Figura1 - Etapas da Revisão Integrativa

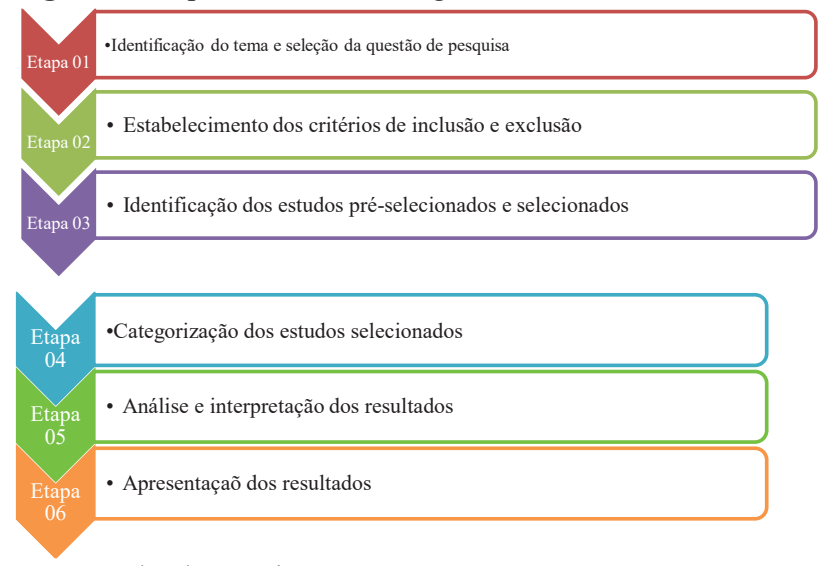

Fonte: Dados da pesquisa. 


\subsubsection{Etapa 1: Identificação do tema e seleção da questão da pesquisa}

A definição da questão que irá nortear a pesquisa, sendo a fase mais importante da revisão, pois determina quais serão os estudos incluídos, os meios adotados para identificação e as informações coletadas a partir de cada estudo. Nesse ínterim se inclui a definição dos participantes, as intervenções a serem avaliadas e os resultados a serem mensurados (WHITTEMORE; KNAFL, 2005).

Utiliza-se a estratégia PICO, acrônimo de população estudada (P), área de interesse (I); comparar ou controle (C), obter resultados através do estudo $(\mathrm{O})$.

Quadro 1 - Elementos da estratégia de PICO. Fortaleza-CearáBrasil, 2020

\begin{tabular}{|c|c|c|}
\hline Acrônico & Definição & Palavras-chave \\
\hline P: População & Pescadores & Pesca \\
\hline I: Interesse & Câncer de Pele & $\begin{array}{l}\text { Câncer de Pele, } \\
\text { Promoção da Saúde }\end{array}$ \\
\hline $\begin{array}{l}\text { C: Controle ou } \\
\text { comparação }\end{array}$ & $\begin{array}{c}\text { Estudos } \\
\text { Correlacionados }\end{array}$ & Estudos, Pesquisas \\
\hline $\begin{array}{l}\text { O: Obter } \\
\text { resultados }\end{array}$ & $\begin{array}{l}\text { Síntese dos riscos e } \\
\text { medidas protetivas }\end{array}$ & $\begin{array}{c}\text { Dados } \\
\text { epidemiológicos/ } \\
\text { Categorias } \\
\text { Temáticas }\end{array}$ \\
\hline
\end{tabular}

Fonte: dados da pesquisa.

A estratégia PICO pode ser usada para formular questões de pesquisa de áreas distintas. Ao definir a pergunta específica, construída adequadamente, há uma maior chance de obter evidências sólidas, focadas na pesquisa, evitando assim buscas desnecessárias (SANTOS; PIMENTA; NOBRE, 2007).

A pergunta norteadora deste estudo foi: quais fatores de risco para a ocorrência de câncer de pele em pescadores? Após a definição do tema do trabalho, o passo seguinte foi a definição dos descritores para a estratégia de busca de pesquisas relacionadas em base de dados (MOREIRA, 2014).

Para a coleta de dados foi realizada uma busca de artigos científicos nas seguintes bases de dados, tais quais: Scientific Eletronic Library Online (SCIELO), Biblioteca Virtual em Saúde (BVS), Literatura Latino-Americana e do Caribe em Ciências da Saúde (LILACS), Base de Dados de Enfermagem (BDENF) e Medical LiteratureAnalysisandRetrieva Online (MEDLINE).

Os descritores, retirados dos Descritores em Ciências da Saúde - DECSassociados à BVS, foram: "Neoplasias Cutâneas", "Promoção da Saúde", associados às palavraschave: "Câncer de Pele, "Pesca" e "Promoção da Saúde". Estes foram combinados entre si, além da utilização do descritor booleano AND.

\subsubsection{Etapa 02: Estabelecimentos de critérios de inclusão e exclusão}

A seleção de amostra para esta revisão contou com os seguintes critérios de inclusão: o período de publicação do estudo: as buscas se restringiram aos estudos publicados nos últimos dez anos (recorte temporal de 2010 a 2020), materiais publicados em Língua Portuguesa e Inglesa traduzidos, disponíveis na íntegra e que possuam alguma relação com a temática do estudo. Já como critérios de exclusão: estudos duplicados e disponíveis de forma parcial ou incompleta.

\subsubsection{Etapa 03: Identificação dos estudos pré-selecionados e selecionados}

Nesta etapa, para a extração de dados a partir dos estudos selecionados se faz necessária a implementação de instrumentais elaborados, capazes de garantir que a totalidade dos dados relevantes seja extraída, dessa forma, minimizando riscos de erros na transcrição, garantindo precisão na checagem das informações e ainda servir de registro, respeitando os critérios de inclusão e exclusão expostos, norteou a busca do material bibliográfico que compôs esta revisão, revelando as evidências científicas acerca da temática.

Quadro 2 - Publicações Científicas disponíveis nas Bases de Dados

\begin{tabular}{|c|c|}
\hline Base de dados & Quantitativo de Artigos \\
\hline BVS & 09 arquivos \\
\hline BDENF & 03 arquivos \\
\hline MEDLINE & 01 artigo \\
\hline SCIELO & 0 artigos \\
\hline LILACS & 06 artigos \\
\hline Total & 19 materiais \\
\hline
\end{tabular}

Fonte: dados da pesquisa.

No que concerne à plataforma BVS, foram encontrados 09 artigos a partir da combinação dos descritores e palavraschave. Já na plataforma SciELO não foram encontrados artigos científicos com os descritores e palavras-chave eleitos. No que tange ao Medline, foi encontrado apenas um artigo científico após busca, enquanto na plataforma BDENF, três publicações emergiram. Na LILACS foi encontrado um total de 06 artigos. Finalmente, após leitura na íntegra do material bibliográfico encontrado e aplicação dos critérios de exclusão estabelecidos, como duplicidade de trabalhos, a amostra final ficou composta por um total de 05 materiais bibliográficos, três artigos e duas dissertações de mestrado, que foram analisados individualmente, sendo expostos posteriormente para discussão.

Utiliza-se uma tabela previamente elaborada para facilitar a visualização dos estudos e favorecer a análise. Inicialmente, se propôs uma leitura geral de cada texto, seguido de leitura seletiva e minuciosa e, por fim, leitura interpretativa e resumo.

Distribuição quantitativa das bibliografias encontradas nas bases de dados: 
Figura 2 - Fluxograma da seleção dos artigos nas bases de dados BVS, SciELO, Lilacs, BDENF e Medline.

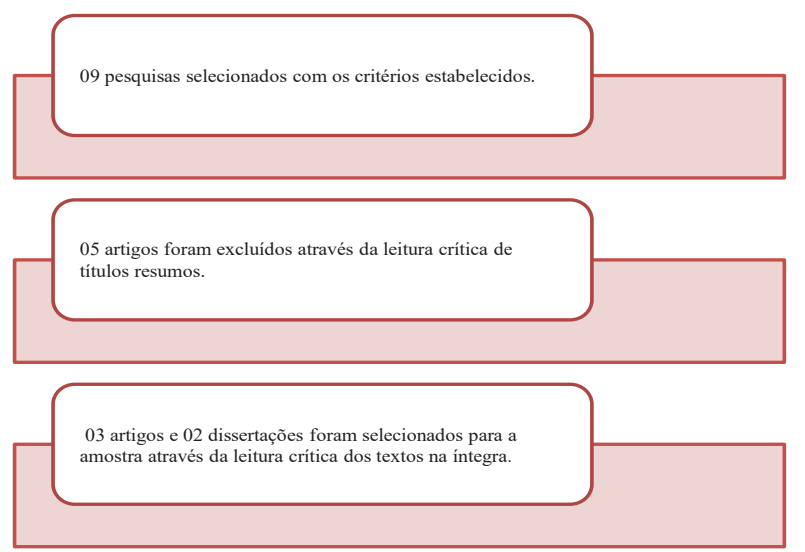

Fonte: dados da pesquisa.

\subsubsection{Etapa 04 e 05: Categorização e Análise dos Estudos selecionados}

Conforme Botelho, De Almeida Cunha e Macedo (2011), a categorização dos estudos pode se basear na incidência, nas características da amostra, bem como na classificação conceitual ou tipo de estudo, que facilite a interpretação dos mesmos. Os estudos foram inseridos em uma tabela e avaliados para classificação dos resultados, conforme recomendado por Moreira (2014). Em seguida, iniciou-se a escrita do documento que consolida e descreve detalhadamente a revisão, sintetizando o conhecimento.

O período da coleta de dados foi Agosto a Dezembro de 2020, no qual os artigos foram selecionados e, posteriormente, analisados.

Quadro 3 - Estudos selecionados após análise

\begin{tabular}{|c|c|c|c|c|c|}
\hline \begin{tabular}{|c|}
$\begin{array}{c}\text { Base de } \\
\text { Dados }\end{array}$ \\
\end{tabular} & \begin{tabular}{|l|} 
Identificação do \\
Estudo (Título) \\
\end{tabular} & Autores & Objetivo da Pesquisa & Definição do Trabalho & Ano \\
\hline Lilacs & \begin{tabular}{|l|} 
Exposição solar \\
e envelhecimento \\
precoce em \\
trabalhadores \\
praianos do \\
município de \\
Salinópolis/PA
\end{tabular} & $\begin{array}{l}\text { Rebelo et } \\
\text { al. }\end{array}$ & 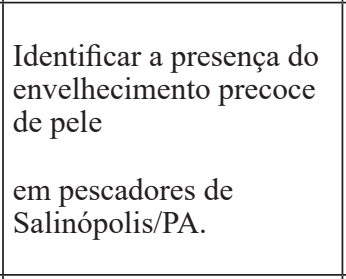 & $\begin{array}{l}\text { Participantes: } 275 \text { pescadores (de fototipo I a VI). } \\
\text { Aplicação de dois questionários: "Protocolo de } \\
\text { Avaliação Facial PAF" e "Hábitos de exposição e } \\
\text { fotoproteção solar". Dados na Tabela } 5 \text { comprovam o } \\
\text { baixo conhecimento sobre os malefícios da radiação } \\
\text { ultravioleta, presença de hábitos inadequados de } \\
\text { exposição solar, como a exposição em horários } \\
\text { indevidos e a não utilização de protetores solares. }\end{array}$ & 2018 \\
\hline BVS & $\begin{array}{l}\text { Câncer de pele: } \\
\text { conhecimento, } \\
\text { práticas e atitudes } \\
\text { de pescadores. }\end{array}$ & $\begin{array}{c}\text { Bushatsky } \\
\text { et al. }\end{array}$ & $\begin{array}{l}\text { Descrever o } \\
\text { conhecimento, as } \\
\text { atitudes e as práticas a } \\
\text { respeito da prevenção } \\
\text { do câncer de pele em } \\
\text { pescadores na Colônia } \\
\text { de Pescadores do Pina, } \\
\text { Recife. }\end{array}$ & $\begin{array}{l}\text { Estudo transversal com } 90 \text { pescadores que se expõem } \\
\text { mais de três horas ao sol durante todo o dia, indicando } \\
\text { uma longa exposição solar. Não utilizam medidas } \\
\text { eficazes para sua proteção, a maior parte protege } \\
\text { apenas o rosto, expondo as outras regiões do corpo ao } \\
\text { sol sem uma medida de proteção. Torna-se evidente } \\
\text { a necessidade de inserção de atividades de caráter } \\
\text { educativo para os pescadores para adoção de métodos } \\
\text { preventivos,assim como para a detecção precoce de } \\
\text { lesões malignas. }\end{array}$ & 2016 \\
\hline BDENF & $\begin{array}{l}\text { Diálogos com } \\
\text { pescadores sobre } \\
\text { o câncer de pele } \\
\text { na perspectiva da } \\
\text { educação popular } \\
\text { em saúde. }\end{array}$ & Moreira. & $\begin{array}{lr}\text { Descrever } & \text { de } \\
\text { conhecimento } & \text { de } \\
\text { pescadores a respeito do } \\
\text { câncer de pele; Discutir } \\
\text { a repercussão da prática } \\
\text { educativa em saúde de } \\
\text { enfermeiras com essa } \\
\text { população, no que tange } \\
\text { a prevenção do câncer de } \\
\text { pele não melanoma }\end{array}$ & $\begin{array}{l}\text { Dissertação qualitativa, do tipo exploratória, com } \\
\text { interface com a pesquisa participante realizada entre } \\
\text { janeiro de } 2013 \text { a novembro de } 2014 \text {. O cenário foi } \\
\text { a Colônia de Pescadores de Jurujuba, localizada em } \\
\text { Niterói/RJ. Os sujeitos foram } 30 \text { pescadores com idade } \\
\text { entre } 25 \text { e } 75 \text { anos, de ambos os sexos.Surgiram três } \\
\text { categorias temáticas: Câncer de pele primeiras palavras; } \\
\text { Câncer de pele medidas de prevenção e Câncer de Pele } \\
\text { modos de ensinar e de aprender. }\end{array}$ & 2015 \\
\hline BDENF & \begin{tabular}{|l|} 
Impacto \\
ambiental, \\
trabalho e saúde \\
de pescadores \\
da Baía de \\
Guanabara RJ, \\
Brasil: a educação \\
pelos pares como \\
estratégia de \\
prevenção.
\end{tabular} & Ribeiro & $\begin{array}{l}\text { Caracterizar as condições } \\
\text { de trabalho e saúde de } \\
\text { pescadores e discutir uma } \\
\text { proposta participativa de } \\
\text { cuidado em saúde a partir } \\
\text { da Educação pelos Pares } \\
\text { com foco nas feridas } \\
\text { cutâneas. }\end{array}$ & $\begin{array}{l}\text { Trata-se de uma pesquisa participante com abordagem } \\
\text { qualitativa sobre o agir educativo participativo da } \\
\text { enfermeira com pescadores artesanais da Baía de } \\
\text { Guanabara-RJ. }\end{array}$ & 2013. \\
\hline BVS & $\begin{array}{l}\text { Práticas do uso } \\
\text { de protetor solar } \\
\text { nos pescadores } \\
\text { da cidade de } \\
\text { Cananéia - SP. }\end{array}$ & $\begin{array}{l}\text { Chimite } \\
\text { et al. }\end{array}$ & $\begin{array}{l}\text { Averiguar o uso do pro- } \\
\text { tetor solar em pescadores } \\
\text { do litoral sul paulista da } \\
\text { cidade de Cananéia. }\end{array}$ & $\begin{array}{l}\text { Os resultados apontaram que a maioria não utiliza o } \\
\text { protetor solar por } \\
\text { falta de conhecimento e devido ao baixo poder } \\
\text { econômico para a compra do mesmo, } \\
\text { enfatizando que tal } \\
\text { procedimento é, portanto, de interesse da saúde } \\
\text { pública. }\end{array}$ & 2017 \\
\hline
\end{tabular}




\subsection{Resultados e Discussão}

Os resultados desta pesquisa confirmam outros estudos que exprimem a alta prevalência de desenvolvimento de neoplasias cutâneas em grupos populacionais especificamente expostos à radiação solar ultravioleta constante e contínua (PETERS; NICOL; DEMERS, 2012; POPIM et al, 2008; CEZAR-VAZ et al., 2015). Algumas variáveis associadas a esse grupo laboral, de trabalhadores expostos à radiação solar ultravioleta foram: a média de idade dos trabalhadores com idade acima de 40 anos de idade, maioria significativa de participantes do sexo masculino e trabalhadores ao ar livre.

Estudos realizados com outros trabalhadores também vulneráveis à radiação, como engenheiros, trabalhadores rurais, hortifrutigranjeiros entre outros profissionais, demonstram que houve avanço nas atitudes preventivas, especificamente, no que diz respeito ao uso de protetor solar após Oficinas de Educação em Saúde (LEE et al., 2014; CEZAR-VAZ et al., 2015), levantando hipóteses positivas quanto ao sucesso de intervenções educativas na população de pescadores.

Estudo recente (RYDZ et al., 2020), idealizado com trabalhadores em Alberta, Canadá, confirmou elevadas taxas de raios ultravioletas em $45 \%$ da amostra (179 participantes ao todo), após medição com uso de dosímetro eletrônico. Resultados semelhantes foram percebidos em trabalho de Borup, Mortensen e Grandahl (2020), na Dinamarca, em que também foi utilizado o dosímetro, na qual participaram 450 trabalhadores ao ar livre por 5 meses.

Chimite et al (2017) encontraram uma parcela dos participantes que fazem uso do protetor solar habitualmente (47\% dos pescadores), todavia somente $10 \%$ reaplicavam a cada duas horas de exposição solar.

A atuação dos Enfermeiros na Atenção Básica à Saúde se mostrou importante, realizando estratégias de educação em saúde, sendo listada como essencial para que esses conhecimentos preventivos alcancem de forma efetiva a população (BUSHATSKY, 2016). Há ainda outras pesquisas que abordam outros profissionais da saúde na atuação junto à população de pesquisadores, como Farmacêuticos, Fisioterapeutas e Cosmetólogos. Por fim, também foram encontradas pesquisas nas quais os pescadores, sob outras vertentes, que não as Ciências da Saúde (RAMIRES; BARRELLA; ESTEVES, 2012).

\section{Conclusão}

Por meio da amostra selecionada se observou que em todos os seis trabalhos havia a constatação de que os pescadores têm hábitos de exposição solar excessiva e não realizam a fotoproteção diariamente no exercício da função laboral, haja vista que se expõem diariamente ao Sol por, no mínimo, 3 horas seguidas, e apesar de terem algum conhecimento e compreensão que a radiação solar possa causar danos sérios à saúde da pele, como as neoplasias cutâneas, a informação é restrita em "ouvir falar", não se convertendo em ações efetivas de fotoproteção. Salienta-se ainda que os equipamentos de proteção individual (EPI) são insuficientes ou ainda inadequados, como uso de bonés em detrimento de chapéus com abas.

Vale reiterar que os cânceres de pele, pelas suas taxas altas de incidência e prevalência, se configuram como uma afetação de Saúde Pública brasileira, entretanto, também se percebem numerosos estudos no âmbito internacional, mostrando a necessidade de mais pesquisas que explorem os cuidados de Atenção à Saúde à população de pescadores que se encontram expostos à radiação solar ultravioleta continuamente.

Dessa forma, ressalta-se a importância da Promoção da Saúde voltada para a população de pescadores visando o autocuidado, com linguagem acessível, próxima à realidade cotidiana dos mesmos, permitindo que sejam instaurados bons hábitos, de forma rotineira e contínua, através do fortalecimento do conhecimento quanto aos processos envolvidos no adoecimento, suscitando mudanças positivas de comportamento cotidiano neste grupo populacional vulnerável ao acometimento de câncer de pele, por trabalharem ao ar livre, mais expostos que a maioria dos trabalhadores em outras profissões.

\section{Referências}

AMERICAN CANCER SOCIETY- ACS. Cancer facts \& figures 2019. Atlanta: American Cancer Society, 2019.

AMERICAN CANCER SOCIETY-ACS. Risk factors and causes of childhood cancer. Atlanta: American Cancer Society, c2019b.

BARDINI, G., LOURENÇO, D., FISSMER, M.C. Avaliação do conhecimento e hábitos de pacientes dermatológicos em relação ao câncer da pele. Arq. Catarin. Med., v.41, n.2, p.56-63, 2012.

BALOGH, T.S. et al. Proteção à radiação ultravioleta: recursos disponíveis na atualidade em fotoproteção. Na. Bras. Dermatol., v.86, n.4, p.732-742, 2011.

BORUP, H.; MORTENSEN, O. S.; GRANDAHL, K. Sex as a risk factor for solar ultraviolet radiation exposure? PhotochemPhotobiol, v.96, n.6, p. 1350-1354, 2020.

BRAY, F. et al. Global cancer statistics 2018: GLOBOCAN estimates of incidence and mortality worldwide for 36 cancers in 185 countries. CA A Cancer J. Clin., v.68, n.6, p.394-424, 2018.

BRAY, F. et al. Planning and developing populations-based cancer registration inlow-and middle-income settings. Lyon: IARC, 2014.

BUSHATSKY, M. et al. Câncer de pele: conhecimento, práticas e atitudes de pescadores. Cogitare Enferm., v. 21, n.1, p. 01-09, 2016.

CEZAR-VAZ, M.R. et al. Câncer de pele em trabalhadores rurais: conhecimento e intervenção de enfermagem. Rev. Esc. Enferm. USP, v. 49, n.4, p.564-571, 2015.

CHIMITE, A. A. H. et al. Práticas do uso de protetor solar nos pescadores da cidade de Cananéia - SP. Rev. Gestão Foco, v. 9, p.402, 2017.

FERLAY, J. et al. Estimating the global cancer incidence and mortality in 2018: GLOBOCAN sources and methods. Int. J. Cancer, v.144, v.8, p.1941-1953, 2020.

GALVÃO, C.M.; SAWADA, N.O.; TREVIZAN, M.A. Revisão 
sistemática: recurso que proporciona a incorporação das evidências na prática da enfermagem. Rev. Latinoam Enferm., v.12, n.3, p.549-56. 2004.

IBGE - Instituto Brasileiro de Geografia e Estatística. Estimativas da população residente para os municípios e para as unidades da federação brasileiros com data de referência em $1^{\circ}$ de julho de 2019. Rio de Janeiro: IBGE, 2019.

INSTITUTO NACIONAL DO CÂNCER JOSÉ ALENCAR GOMES DA SILVA. Estimativa 2014: incidência de câncer no Brasil. Rio de Janeiro: INCA; 2015.

INSTITUTO NACIONAL DE CÂNCER JOSÉ ALENCAR GOMES DA SILVA. Tipos de câncer. Rio de Janeiro: INCA, 2019.

LAGES, R.B. et al. Detecção precoce do câncer de pele: experiência de campanha de prevenção no Piauí-Brasil. Rev. Bras. Promoção Saúde, 2012

LEE, C. et al. The impact of sun solutions educational interventions on select health belief model constructs. Workplace
Health Saf. v.62, n.2, p.70-9, 2014.

PETERS, C.E; NICOL, A.M, DEMERS, P.A. Prevalence of exposure to solar ultraviolet radiation (UVR) on the job in Canada. Can. J. Public. Health., v.103, n.3, p. 223-6, 2012.

POPIM, R. C. et al. Câncer de pele: uso de medidas preventivas e perfil demográfico de um grupo de risco na cidade de Botucatu. Ciênc. saúde coletiva, v.13, n.4, p. 71-83, 2008.

RYDZ, E. et al. Solar ultraviolet tradiation exposure among outdoor workers in Alberta. Canada Environ Res, v.189, p. 109902, 2020.

RAMIRES, M.; BARRELLA, W.; ESTEVES, A.M. Caracterização da pesca artesanal e o conhecimento pesqueiro local no vale do ribeira e litoral sul de São Paulo. Revista Ceciliana, v. 4, n.1, 37-43, 2012.

RIOS, A.O.; REGO, R.C.F.; PENA, P.G.L. Doenças em trabalhadores da pesca. Rev. Baiana Saúde Pública, v.35, n.1, p. 175-88. 2011. 